\title{
Enhancement of neuroprotective activity of Sagunja-tang by fermentation with lactobacillus strains
}

\author{
Nam-Hui Yim ${ }^{\dagger}$, Min Jung Gu+, Hee Ra Park, Youn-Hwan Hwang and Jin Yeul Ma
}

\begin{abstract}
Background: Sagunja-tang (SGT) is widely used in traditional herbal medicine to treat immune system and gastrointestinal disorders and reportedly has protective effects against inflammation, cancer, and osteoporosis. In this study, we fermented SGT with different Latobacillus strains and investigated the change in phytochemical compositions in SGT and enhancement of it neuroprotective effects in SH-SY5Y human neuroblastoma.
\end{abstract}

Methods: Marker components, including ginsenoside $\mathrm{Rg}_{1}$, glycyrrhizin, liquiritin, liquiritigenin, atractylenolide I, atractylenolide II, atractylenolide III, and pachymic acid, in SGT, were qualitatively and quantitatively analyzed using high-performance liquid chromatography-diode array detection and liquid chromatography-mass spectrometry. SGT was fermented with eight different Lactobacillus strains to yield eight fermented SGTs (FSGTs). The conversion efficiencies of SGT marker components were determined in each FSGT. To detect the protective effect of SGT and FSGT, reactive oxygen species (ROS) assay and mitochondrial membrane potentials (MMPs) assay were performed in SH-SY5Y cells.

Results: Compared with the other FSGTs, SGT166, i.e., SGT fermented with L. plantarum 166, had high conversion efficiency, as indicated by increased amounts of glycyrrhizin, liquiritigenin, and atractylenolides I-III. In SH-SY5Y cells, protection against cell death induced by $\mathrm{H}_{2} \mathrm{O}_{2}$ and etoposide was high using SGT166 and very low using SGT. Furthermore, ROS production and mitochondrial membrane potential disruption in SH-SY5Y cells were markedly suppressed by SGT166 treatment, which demonstrated that inhibition of ROS generation may be one of the neuroprotective mechanisms of SGT166.

Conclusions: This study demonstrated that fermentation of SGT with L. plantarum 166 enhanced suppression of oxidative stress and MMP loss. This enhanced neuroprotective effect was thought to be caused by the conversion of SGT phytochemicals by fermentation. SGT166 shows potential for treating neurological damage-related diseases.

Keywords: Sagunja-tang, Neuroprotective effect, Neuroblastoma, Lactobacillus plantarum 166, Phytochemicals

\section{Background}

Sagunja-tang (SGT; Sijunzi-tang in Chinese and Shikunshi-to in Japanese) is a famous traditional herbal medicine in eastern Asia that has been used to treat spleen deficiency and gastrointestinal disorders, such as vomiting, diarrhea, and ulcer [1]. Recently, SGT has been shown to confer a variety of pharmacological activities, including anti-inflammation, anti-cancer, and

\footnotetext{
* Correspondence: jyma@kiom.re.kr

${ }^{\dagger}$ Nam-Hui Yim and Min Jung Gu contributed equally to this work. Korean Medicine (KM) Application Center, Korea Institute of Oriental Medicine (KIOM), 70 Cheomdan-ro, Dong-gu, Daegu 701-300, Republic of Korea
}

(c) The Author(s). 2018 Open Access This article is distributed under the terms of the Creative Commons Attribution 4.0 International License (http://creativecommons.org/licenses/by/4.0/), which permits unrestricted use, distribution, and reproduction in any medium, provided you give appropriate credit to the original author(s) and the source, provide a link to the Creative Commons license, and indicate if changes were made. The Creative Commons Public Domain Dedication waiver (http://creativecommons.org/publicdomain/zero/1.0/) applies to the data made available in this article, unless otherwise stated. anti-ulceration activities and regulation of bone metabolism [2-5]. SGT is composed of ginseng radix, atractylodis rhizoma, poria sclerotium, and glycyrrhizae radix at a ratio of 1:1:1:1, which themselves contain various phytochemicals, including ginsenoside (ginseng radix), atractylenolides I-III (atractylodis rhizoma), pachyman and pachymic acid (poria sclerotium), liquiritin, liquiritigenin, and glycyrrhizin (glycyrrhizae radix). Recently, Kang et al. investigated simultaneous analytical methods suitable for quantifying the major compounds in SGT [6]. In our study, their established method was modified 
and applied to analyze seven marker compounds in SGT and fermented SGT (FSGT).

Fermentation with probiotics, such as Lactobacillus spp., Bifidobacterium spp., and Saccharomyces spp., has been shown to affect the conversion of phytochemical composition and improve the therapeutic effects of some herbal medicines [7-9]. The results of several studies have suggested that fermentation of natural medicines with Lactobacillus increases the concentrations of bioactive components, especially antioxidants, which influence bioactivities, including antiinflammatory, antiosteoporotic, and anticancer activities [10-13]. Our previous study identified the antiamnesic effects of Sipjeondaebo-tang fermented with Lactobacillus in a scopolamine-induced amnesia mouse model [14].

In neuronal cells, oxidative stress causes mitochondrial dysfunctions, which is involved in apoptotic cell death. Reactive oxygen species (ROS) and reactive nitrogen species (RNS) play an important role as regulatory mediators in physiological system, however, the high concentrations of these species are involved in cellular damage, which causes the neurodegenerative diseases, including Alzheimer's disease (AD), Parkinson's disease (PD), Mutiple Sclerosis (MS) and Amyotrophic lateral sclerosis (ALS) [15-19]. Especially, ROS are active in the brain and neuronal tissue and generally lead to neuronal damage and apoptotic cell death induced by intracellular micro-environmental changes [20]. Hence, inhibiting ROS generation can be a useful way to protect normal neuronal cells from damage or death that leads to neurodegenerative diseases.

In the present study, we investigated the conversion of phytochemicals caused by fermentation of eight batches of SGT with eight strains of Lactobacillus. We then assessed the neuroprotective effects of SGT alone and of SGT fermented with L. plantarum 166 (SGT166) in SH-SY5Y human neuroblastoma.

\section{Methods}

\section{Chemicals and reagents}

Ginsenoside $\mathrm{Rg}_{1}$ and glycyrrhizin were purchased from the Korea Food and Drug Administration and Tokyo Chemical Industry Co., Ltd. (Tokyo, Japan), respectively. Liquiritin, liquiritigenin, atractylenolide I, atractylenolide II, atractylenolide III, and pachymic acid were purchased from Faces Biochemical Co., Ltd. (Wuhan, China). The purities of these seven reference standards were $>98 \%$, and their structures are shown in Fig. 1. Trifluoroacetic acid (TFA) and formic acid were purchased from Sigma-Aldrich Co. (St. Louis, MO, USA). Acetonitrile of high-performance liquid chromatography (HPLC) grade was obtained from J.T. Baker Inc. (Philipsburg, NJ, USA), and deionized water was prepared using an ultrapure water production apparatus (Millipore, Billerica, MA, USA).

\section{Preparation of SGT and FSGT}

All herbal constituents of SGT, including ginseng radix (KMAC112), atractylodis rhizome (KMAC059), poria sclerotium (KMAC061), and glycyrrhizae radix (KMAC003), were purchased from the Korea Medicinal Herbs Association (Yeongcheon, Korea). The origins of the samples were confirmed taxonomically by Prof. Ki Hwan Bae of the College of Pharmacy, Chungnam National University. All voucher specimens were deposited in the herbal bank of the Korean Medicine Application Center, Korea Institute of Oriental Medicine. Four kinds of medicinal herbs $(2 \mathrm{~kg}$; Table 1$)$ were extracted into $20 \mathrm{~L}$ of water for $3 \mathrm{~h}$ using a COSMOS-660 extractor (Kyungseo Machine Co., Incheon, Korea). The extract was filtered through standard testing sieves $(150 \mu \mathrm{m})$ and freeze-dried to yield SGT extract powder $(240 \mathrm{~g})$. To ferment SGT, the following eight bacterial strains were used to generate eight batches: Lactobacillus rhamnosus KFRI127, L. zeae KFR129, L. rhamnosus KFRI144, L. acidophilus KFRI150, L. fermentum KFRI162, L. plantarum KFRI166, L. acidophilus KFRI217, and L. helveticus KFRI341. The strains were obtained from the Korea Food Research Institute (Seongnam, Korea) and were cultured in de Man, Rogsa, and Sharpe broth at $37^{\circ} \mathrm{C}$ for $24 \mathrm{~h}$ and reinoculated to each broth under the same conditions. The solutions of SGT extract were adjusted to $\mathrm{pH} 8.0$ with $1 \mathrm{M} \mathrm{NaOH}$ and autoclaved for $15 \mathrm{~min}$ at $121{ }^{\circ} \mathrm{C}$ for use as the culture media in the bacterial fermentations. The solutions were inoculated with $5 \mathrm{~mL}$ of the inoculum $(1 \% v / \mathrm{v}, 2 \times$ $10^{9} \mathrm{CFU} / \mathrm{mL}$ ). The inoculated samples were incubated at $37{ }^{\circ} \mathrm{C}$ for $48 \mathrm{~h}$. The FSGT was lyophilized at $4{ }^{\circ} \mathrm{C}$ after filtering through a $60-\mu \mathrm{m}$ nylon net filter (Millipore, Billerica, Mass, USA).

\section{Preparation of standard solutions and analytical samples} Individual stock solutions of liquiritin $(400 \mu \mathrm{g} / \mathrm{mL})$, ginsenoside $\operatorname{Rg}_{1}(500 \mu \mathrm{g} / \mathrm{mL})$, liquiritigenin $(200 \mu \mathrm{g} / \mathrm{mL})$, glycyrrhizin $(900 \mu \mathrm{g} / \mathrm{mL})$, atractylenolide I $(200 \mu \mathrm{g} / \mathrm{mL})$, atractylenolide II $(200 \mu \mathrm{g} / \mathrm{mL})$, and atractylenolide III $(200 \mu \mathrm{g} / \mathrm{mL})$ dissolved in methanol were mixed and diluted serially to five concentrations for use in generating calibration curves. Quality control samples for method validation were diluted serially to three concentrations. The SGT and FSGTs for quantitative analysis were dissolved at a concentration of $20 \mathrm{mg} / \mathrm{mL}$ in methanol. The samples were filtered through a $0.2 \mu \mathrm{m}$ membrane filter (Whatman International Ltd., Maidstone, UK) and stored at $4{ }^{\circ} \mathrm{C}$ before use. 
<smiles>O=C1C[C@H](c2ccc(OC(OCCCO)C(O)C(O)CO)cc2)Oc2cc(O)ccc21</smiles>

Liquiritin<smiles>O=c1cc(-c2ccc(O)cc2)oc2cc(O)ccc12</smiles>

Liquiritigenin

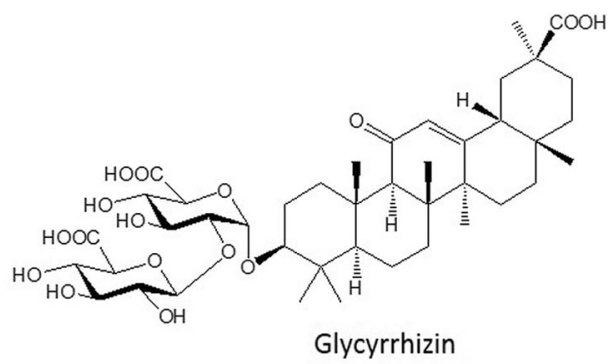

Glycyrrhizin

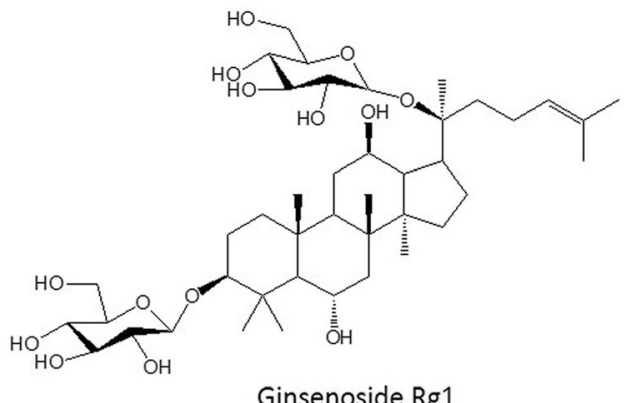

Ginsenoside Rg1<smiles>C=C(CC[C@H](C(=O)O)[C@@]1(C)[C@H](O)C[C@]2(C)C3=C(CC[C@]21C)[C@@]1(C)CC[C@H](OC(C)=O)C(C)(C)[C@]31C)C(C)C</smiles>

Pachymic acid<smiles>C=C1CCC[C@]2(C)C[C@H]3OC(=O)C(C)=C3C[C@]12C</smiles>

Atractylenolide I<smiles>C=C1CCC[C@]2(C)C=C3OC(=O)C(C)=C3C[C@H]12</smiles>

Atractylenolide II<smiles>C=C1CCC[C@]2(C)C[C@]3(O)OC(=O)C(C)=C3C[C@H]12</smiles>

Atractylenolide III

Fig. 1 Chemical structures of the eight components in SGT

\section{Chromatographic conditions}

The analysis was performed on an HPLC system (Hitachi High-Technologies Co., Tokyo, Japan), consisting of a pump (L-2130), an auto sampler (L-2200), a column oven (L-2350), and a diode array UV/VIS detector (L-2455). The system control and data analyses were executed by an EZchrom Elite software (version 3.3.1a) system. The separation was performed on a RS tech $\mathrm{C}_{18}$ column $(5 \mu \mathrm{m}, 250 \times 4.60 \mathrm{~mm})$ with a flow rate of $1.0 \mathrm{~mL} / \mathrm{min}$ and a column oven temperature of $40{ }^{\circ} \mathrm{C}$. The mobile phase consisted of $0.1 \%$ trifluoroacetic acid in water (A) and acetonitrile (B). To improve the chromatographic separation capacity, the gradient elution system was performed as follows: $90-65 \%$ A, 0-25 min;

Table 1 Composition of Sagunja-tang (SGT) preparation

\begin{tabular}{lll}
\hline Herbal medicine & Amount $(\mathrm{g})$ & Ratio (\%) \\
\hline Ginseng Radix & 500 & 25 \\
Atractylodis Rhizoma & 500 & 25 \\
Poria Sclerotium & 500 & 25 \\
Glycyrrhizae Radix & 500 & 25 \\
Total & 2000 & 100 \\
\hline
\end{tabular}

65-55\% A, 25-40 min; 55-30\% A, 40-55 min; 30\% A, 55-65 min. The sample injection volume was $20 \mu \mathrm{L}$.

Liquid chromatography/mass spectrometry (LC/MS) analysis was performed on a Dionex UltiMate 3000 system (Dionex Corp., Sunnyvale, CA, USA) equipped with a Thermo Q-Exactive mass spectrometer (Thermo Fisher Scientific, Bremen, Germany). The separation was performed on an Acclaim RSLC 120 C18 column (150 × $2.1 \mathrm{~mm}, 2.2 \mu \mathrm{m}$, Dionex Corp.). The mobile phase consisted of acetonitrile (reservoir A) and $0.1 \% \mathrm{v} / \mathrm{v}$ formic acid in water (reservoir B). Gradient elution was as follows: $3 \% \mathrm{~A}, 0.0-2.0 \mathrm{~min} ; 3.0-40.0 \% \mathrm{~A}, 2.0-15.0 \mathrm{~min}$; 40.0-95.0\% A, $15.0-23.0$ min; $95.0-95.0 \%$ A, 23.0$24.0 \mathrm{~min} ; 95.0-100 \% \mathrm{~A}, 15.0-17.0 \mathrm{~min} ; 5 \%$ isocratic A, $17.0-22.0 \mathrm{~min}$. The flow rate was $0.4 \mathrm{~mL} / \mathrm{min}$. The operation conditions in the MS analysis were set as follows: ionization mode, positive; spray voltage, $4.0 \mathrm{kV}$; capillary temperature, $320{ }^{\circ} \mathrm{C}$; sheath gas pressure, 40 arbitrary units; auxiliary gas pressure, 10 arbitrary units; ion scans, $100-1500 \mathrm{~m} / \mathrm{z}$; resolution of MS scans, 70,000. The SGT or SGT166 $(10 \mathrm{mg} / \mathrm{mL})$ and mixture of authentic standards $(10 \mu \mathrm{g} / \mathrm{mL})$ were prepared in methanol and filtered through a $0.22 \mu \mathrm{m}$ filter membrane before injecting $5-\mu \mathrm{L}$ aliquots for HPLC-MS analysis. 


\section{Method validation}

Method validation for selectivity, linearity, limit of detection (LOD), limit of quantification (LOQ), precision and accuracy, and recovery in the present study was executed according to the guideline of International Conference on Harmonization (ICH).

The standard calibration curve for the linearity assay was prepared with five different concentrations of diluted standard solutions and performed in triplicate independently. The calibration curves were constructed by plotting the value of the peak versus the concentration of each analyte. The lower LOD and lower LOQ for each analyte were determined on the basis of signal-to-noise ratios $(\mathrm{S} / \mathrm{N})$ of 3.3 and 10 , respectively.

The precision of the analytical method was evaluated by intra- and inter-day tests. Standard solutions at three different concentrations (low, medium, and high) were analyzed. The intra-day test was performed by analyzing a mixed standard solution in five replicates during one day. The inter-day test was performed by analyzing five replicates of the same standard solution on each of three consecutive days. Precision was expressed as relative standard deviation (RSD, \%), which is generally acceptable within $3 \%$. The related equation was as follows: RSD $(\%)=[$ standard deviation (SD)/mean measured amount] $x 100$. The recovery tests were performed to evaluate the accuracy of the method. The recoveries of analytes were determined by adding three different concentrations of each standard solution into the SGT solution $(10 \mathrm{mg} / \mathrm{mL})$ in triplicate. Recovery (\%) was calculated according to the following equation: Recovery $(\%)=[$ found amount - original amount $] /$ spiked amount $\mathrm{x} 100$.

\section{Cell culture}

SH-SY5Y cells, a human neuroblastoma-derived cell line with neuron-like characteristics, were provided by Prof. Jaewon Lee, Pusan National University, Korea. The cells were differentiated into neuron-like phenotype by induction of retinoic acid and cultured with RPMI 1640 media (Lonza, Walkersville, MD, USA) with heat-inactivated $10 \%$ fetal bovine serum (HyClone Laboratories, Utah, USA), $2 \mathrm{mM}$ glutamine, and 1\% penicillin/streptomycin antibiotic mixture (Corning Incorporated, NY, USA) in a humidified $5 \% \mathrm{CO}_{2}$ incubator at $37{ }^{\circ} \mathrm{C}$.

\section{Cell viability analysis}

Cells $\left(1 \times 10^{4}\right.$ cells $\left./ \mathrm{mL}\right)$ were seeded into 96 -well plates. After $24 \mathrm{~h}$, the cells were pretreated with different concentrations of samples ( 250 and $500 \mu \mathrm{g} / \mathrm{mL}$ ) for $6 \mathrm{~h}$ and co-treated with $50-\mu \mathrm{M}$ of the toxicants $\mathrm{H}_{2} \mathrm{O}_{2}$ and etoposide for $24 \mathrm{~h}$. After treatment, cell viability was analyzed using a Cell Counting Kit-8 (CCK-8) solution
(Dojindo Laboratories, Kumamoto, Japan). Color intensity was measured at $450 \mathrm{~nm}$ using ELISA microplate reader.

\section{ROS production}

ROS products were measured using a fluorogenic dye 2 ', $7^{\prime}$-dichlorodihydrofluorescein. diacetate $\left(\mathrm{H}_{2}\right.$-DCFDA), which is oxidized by intracellular ROS. Cells $\left(5 \times 10^{3}\right.$ cells/ well) were seeded into black 96-well plates and pretreated with vehicle or samples for $6 \mathrm{~h}$, treated with $50 \mu \mathrm{M}$ $\mathrm{H}_{2}$-DCFDA for $30 \mathrm{~min}$, and then incubated with $50 \mu \mathrm{M}$ $\mathrm{H}_{2} \mathrm{O}_{2}$ for $30 \mathrm{~min}$. The cells were washed with phosphate-buffered saline, and fluorescent compounds were detected using a fluorescence microplate reader (SpectraMax i3; Molecular Devices, CA, USA) with excitation and emission wavelengths of $495 \mathrm{~nm}$ and $529 \mathrm{~nm}$, respectively.

\section{Mitochondrial membrane potentials (MMPs) assay}

Cells were seeded into a confocal dish (coverglass-bottom dish). After pretreatment with vehicle or samples for $6 \mathrm{~h}$, the cells were co-treated with $100 \mu \mathrm{M} \mathrm{H}_{2} \mathrm{O}_{2}$ for $1 \mathrm{~h}$. The cells were further incubated with JC-1 (chloride salt; Biotium, Hayward, CA, USA) staining solution $(5 \mu \mathrm{g} / \mathrm{mL})$ at $37{ }^{\circ} \mathrm{C}$ for $15 \mathrm{~min}$ and rinsed with culture media. MMPs were estimated by measuring the fluorescence of free JC-1 monomers (green) to JC-1 aggregates in mitochondria (red) as observed through a Nikon ECLIPSE TE2000-U microscope (Nikon Instruments Inc., Tokyo, Japan), and the quantification of the red and green fluorescence intensity ratio in individual cells was performed using Nikon NIS-Elements microscope imaging software.

\section{Statistical analysis}

Data are presented as means \pm SDs. Statistically significant differences between vehicle, toxicants, and sample treated cells were calculated by one-way analysis of variance with Dunnett's test. The analyses were performed using GraphPad PRISM software * (GraphPad PRISM software Inc., Version 5.03, CA, USA). Values of $p<0.05$ were considered as indicating statistical significance.

\section{Results}

\section{Optimization of chromatographic conditions}

Simultaneous quantitative and qualitative analyses of seven compounds in SGT were performed using HPLCDAD and LC/MS, respectively. To improve the chromatographic separation capacity, $0.1 \%$ TFA $(v / v)$ in water (A) and acetonitrile (B) were used as mobile phases in a gradient elution system. Furthermore, analyte resolution was found to be better with acetonitrile than with methanol. The present chromatographic conditions were used to establish the specific HPLC retention times $\left(t_{R}\right)$ and UV detection wavelengths for the seven standard 
compounds, which were used to identify the seven compounds in SGT and in one of the Lactobacillus-fermented SGT batches (SGT166). As shown in Fig. 2, the retention times of liquiritin, ginsenoside $\mathrm{Rg}_{1}$, liquiritigenin, glycyrrhizin, atractylenolide III, atractylenolide II, and atractylenolide I in the standard mixture were $15.25(220 \mathrm{~nm})$,
$22.20(200 \mathrm{~nm}), 23.91(220 \mathrm{~nm}), 36.66(254 \mathrm{~nm}), 44.76$ $(220 \mathrm{~nm}), 52.68(220 \mathrm{~nm})$, and $57.15(275 \mathrm{~nm})$ minutes, respectively. Under the same conditions, the retention times ( $\mathrm{min}$ ) of the observed components were 15.28 (liquiritin), 22.12 (ginsenoside $\operatorname{Rg}_{1}$ ), 23.95 (liquiritigenin), 36.59 (glycyrrhizin), 44.74 (atractylenolide III), 52.67

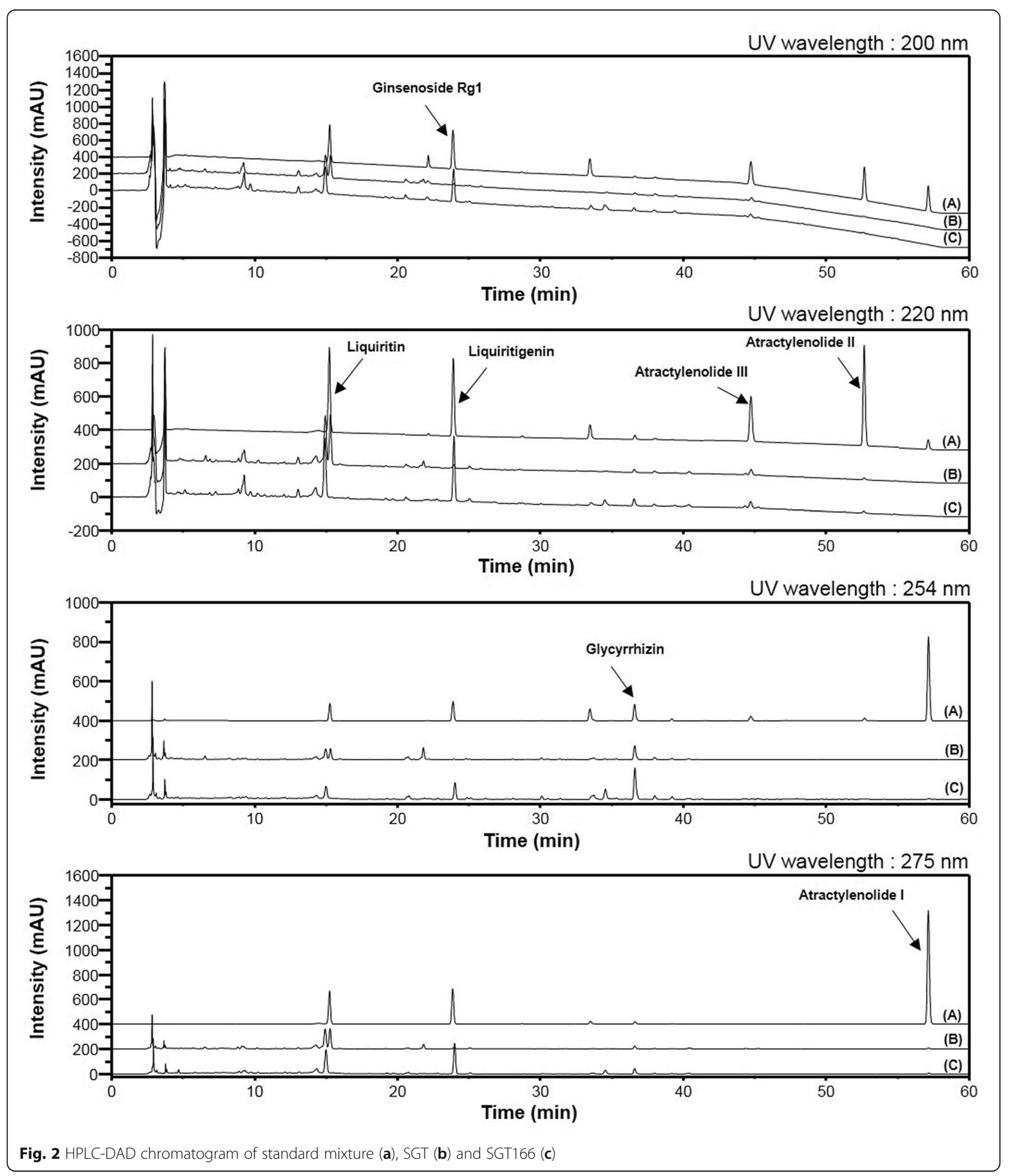


(atractylenolide II), and 57.14 (atractylenolide I) in SGT and 15.22 (liquiritin), 22.10 (ginsenoside $\operatorname{Rg}_{1}$ ), 23.95 (liquiritigenin), 36.55 (glycyrrhizin), 44.71 (atractylenolide III), 52.64 (atractylenolide II), and 57.11 (atractylenolide I) in SGT166. Pachymic acid as a standard compound of poria sclerotium was not detected in our optimized chromatographic method; therefore, pachymic acid was identified by LC/MS analysis (Fig. 3). In positive-ion mode, molecular ions for each compound in the standard mixture were observed at $\mathrm{m} / \mathrm{z} 419.133[\mathrm{M}+\mathrm{H}]^{+}$for liquiritin, $\mathrm{m} / \mathrm{z} 257.080[\mathrm{M}+\mathrm{H}]^{+}$for liquiritigenin, $\mathrm{m} / \mathrm{z}$ 823.481 [M $+\mathrm{Na}]^{+}$for ginsenoside Rg1, m/z $823.411[\mathrm{M}+\mathrm{H}]^{+}$for glycchirizine, $\mathrm{m} / \mathrm{z} 231.137\left[[\mathrm{M}+\mathrm{H}]^{+}\right.$for atractylenolide I, $\mathrm{m} / \mathrm{z} 233.153[\mathrm{M}+\mathrm{H}]^{+}$for atractylenolide II, $\mathrm{m} / \mathrm{z} 249.148$ $[\mathrm{M}+\mathrm{H}]^{+}$for atractylenolide III, and $\mathrm{m} / \mathrm{z} 529.388[\mathrm{M}+$ $\mathrm{H}]^{+}$for pachymic acid. Under the same conditions, molecular ions for each components were detected at $\mathrm{m} / \mathrm{z}$ 419.132 $[\mathrm{M}+\mathrm{H}]^{+}$for liquiritin, $\mathrm{m} / \mathrm{z} 257.080[\mathrm{M}+\mathrm{H}]^{+}$for liquiritigenin, $\mathrm{m} / \mathrm{z} 823.479[\mathrm{M}+\mathrm{Na}]^{+}$for ginsenoside Rg1, m/z $823.409[\mathrm{M}+\mathrm{H}]^{+}$for glycchirizine, $\mathrm{m} / \mathrm{z}$ $231.137\left[[\mathrm{M}+\mathrm{H}]^{+}\right.$for atractylenolide $\mathrm{I}, \mathrm{m} / \mathrm{z} 233.153$ $[\mathrm{M}+\mathrm{H}]^{+}$for atractylenolide II, m/z $249.147[\mathrm{M}+\mathrm{H}]^{+}$ for atractylenolide III, and $\mathrm{m} / \mathrm{z} 529.387[\mathrm{M}+\mathrm{H}]^{+}$for pachymic acid in SGT; m/z $419.132[\mathrm{M}+\mathrm{H}]^{+}$for liquiritin, $\mathrm{m} / \mathrm{z} 257.080[\mathrm{M}+\mathrm{H}]^{+}$for liquiritigenin, $\mathrm{m} / \mathrm{z}$ $823.479[\mathrm{M}+\mathrm{Na}]^{+}$for ginsenoside Rg1, m/z 823.409 $[\mathrm{M}+\mathrm{H}]^{+}$for glycchirizine, $\mathrm{m} / \mathrm{z} 231.137\left[[\mathrm{M}+\mathrm{H}]^{+}\right.$for atractylenolide I, m/z $233.153[\mathrm{M}+\mathrm{H}]^{+}$for atractylenolide II, $\mathrm{m} / \mathrm{z} 249.148[\mathrm{M}+\mathrm{H}]^{+}$for atractylenolide III, and $\mathrm{m} / \mathrm{z} 529.388[\mathrm{M}+\mathrm{H}]^{+}$for pachymic acid in SGT166 (Additional file 1: Table S1).

\section{Analytical methods validation}

To establish the calibration curve, mixed standard solutions of five different concentrations were analyzed in triplicate. Calibration curves were generated by plotting chromatographic peak area as a function of analyte concentration. Linear regressions of these data were expressed as $Y=A x+B$, where $A$ is the slope of the calibration curve, $B$ is the $y$-intercept of the calibration curve, $x$ is the concentration of marker components, and $Y$ is the peak area. Correlation coefficients $\left(R^{2}\right)$ were calculated to assess linearity. The calibration data of each standard compound showed good linearity $\left(R^{2}>0.9999\right)$. The LOD and LOQ were determined at signal-to-noise $(\mathrm{S} / \mathrm{N})$ ratios of 3 and 10 , respectively. The LOD was between $0.002 \mu \mathrm{g} / \mathrm{mL}$ and $0.023 \mu \mathrm{g} / \mathrm{mL}$, and the LOQ was between $0.006 \mu \mathrm{g} / \mathrm{mL}$ and $0.069 \mu \mathrm{g} / \mathrm{mL}$ (Additional file 1: Table S2). The RSD (\%) was $<3 \%$, which indicated acceptable precision. The RSDs of the intra- and inter-day assays were between 0.02 and $1.80 \%$ and between 0.02 and $1.90 \%$, respectively, with accuracy between 97.72 and $102.20 \%$ for the intra-day assay and between 98.11 and $103.06 \%$ for the inter-day assay. Accuracy was determined by a recovery test with an appropriate amount of SGT $(10 \mathrm{mg} / \mathrm{mL})$ that had been spiked with three different (low, medium, and high) quantities of authentic standards. The amounts of each of the seven compounds in the spiked SGT were calculated from the corresponding calibration curve. The results showed good accuracy with an overall recovery between 96.22 and $104.09 \%$ for the compounds concerned. These results demonstrated that the developed HPLC method was sufficiently accurate and sensitive for simultaneous quantitative evaluation of seven components from SGT (Additional file 1: Tables S3 and S4).

\section{Phytochemical characterization of SGT and FSGTs}

The developed HPLC-based analytical method was applied to the simultaneous quantitation of seven components, including liquiritin, ginsenoside $\mathrm{Rg}_{1}$, liquiritigenin, glycyrrhizin, atractylenolide III, atractylenolide II, and atractylenolide I, in SGT and its FSGTs. Among them, the amounts of ginsenoside $\mathrm{Rg}_{1}$ in SGT and the FSGTs were lower than the LOQ even though $\mathrm{Rg}_{1}$ was observed in HPLC chromatograms of SGT and FSGTs (Fig. 2). Therefore, it was recorded as not detected (nd). Except for ginsenoside $\mathrm{Rg}_{1}$, the six components in SGT and the FSGTs were identified by comparing their retention times and UV absorbances with those of the standard compounds. The contents of liquiritigenin, glycyrrhizin, and atractylenolide I-III were increased, whereas the content of
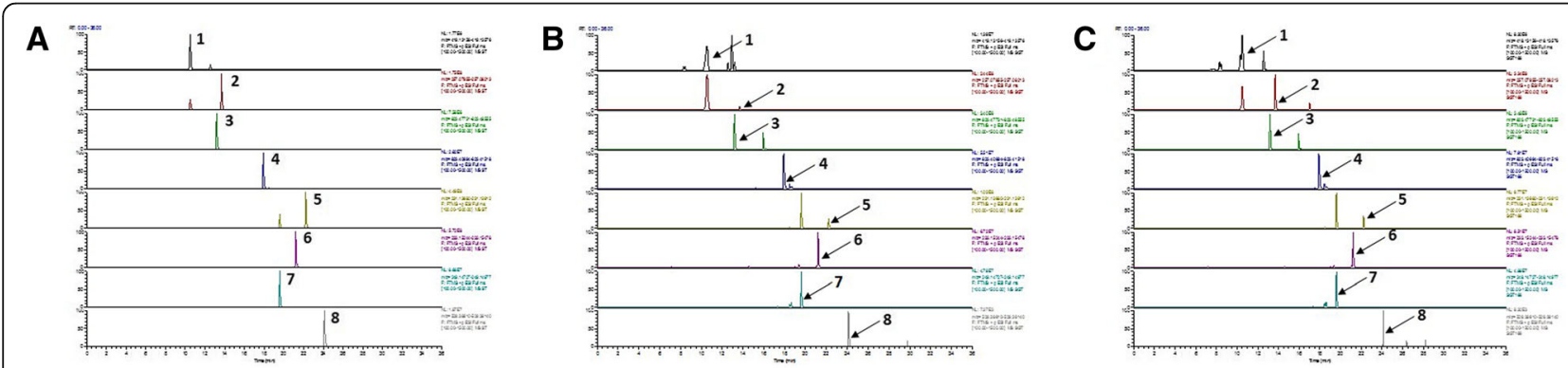

Fig. 3 Extracted ion chromatogram (EIC) of standard mixture (a), SGT (b) and SGT166 (c) using UPLC-ESI-MS analysis. 1, Liquiritin; 2, Liquiritigenin; 3, Ginsenoside Rg 1 ; 4, Glycyrrhizin; 5, Atractylenolide I; 6, Atractylenolide II; 7, Atractylenolide III; 8, Pachymic acid 
liquiritin was decreased in the FSGTs relative to that of the SGT. These results indicate that the detected chemical components could be used as biomarkers for studying SGT and FSGTs.

In this study, following fermentation by all eight kinds of Lactobacillus strains, the conversion between liquiritin and liquiritigenin, an aglycone form of liquiritin, was clearly confirmed: the amount of liquiritigenin increased between 1275.84 and $1399.69 \%$, and the amount of liquiritin decreased by 97.83 to $98.90 \%$. Furthermore, glycyrrhizin contents in SGT was markedly changed by fermentation: the amount of glycyrrhizin increased between 199.44 and $279.28 \%$ (Table 2). In this study, fermentation of SGT by $L$. plantarum 166 (SGT166) showed distinct component changes among the eight kinds of Lactobacillus strains. As presented in Table 3, the conversion rates of liquiritigenin (1399.69\%), glycyrrhizin (279.28\%), atractylenolide III (29.96\%), atractylenolide II (5.98\%), and atractylenolide I (36.67\%) in SGT166 were higher than those in SGT (100\%).

\section{Comparison of the protective effects of SGT and SGT116 against toxicant-induced intracellular oxidative stress and disruption of MMPs in SH-SY5Y cells}

Human neuroblastoma SH-SY5Y cells are widely used as an in vitro model in neuroscience research, including studies of neurobiology, neuronal differentiation, and neuroprotective events [21]. To determine the neuroprotective effects of SGT and SGT166, SH-SY5Y cells were treated with SGT or SGT166 at $250 \mu \mathrm{g} / \mathrm{mL}$ and $500 \mu \mathrm{g} / \mathrm{mL}$ for $24 \mathrm{~h}$, respectively. The treatment with SGT or SGT166 had no significant cytotoxicity on SH-SY5Y cells; therefore, these concentrations were used in further studies (data not shown).

To access the neuroprotective effects of SGT or SGT166, $\mathrm{H}_{2} \mathrm{O}_{2}$ and etoposide were separately used to treat SH-SY5Y cells as a toxicant to induce ROS generation. The cells were pretreated with SGT or SGT166 for $6 \mathrm{~h}$ and then separately exposed to $50 \mu \mathrm{M} \mathrm{H} \mathrm{H}_{2} \mathrm{O}_{2}$ and $50 \mu \mathrm{M}$ etoposide for $24 \mathrm{~h}$. CCK analysis showed that SGT166 significantly protected toxicant-induced SH-SY5Y cell loss, whereas SGT had a much lesser effect (Fig. 4). To further investigate the effect on endogenous ROS in SH-SY5Y cells, SGT and SGT166 were treated separately with $50 \mu \mathrm{M} \mathrm{H} \mathrm{O}_{2}$ or $50 \mu \mathrm{M}$ etoposide. After $24 \mathrm{~h}$, the levels of intracellular ROS were measured using $\mathrm{H}_{2}$-DCFDA, a fluorescent dye oxidized to fluorescent DCF by ROS. Toxicant treatment caused a marked increase in intracellular ROS generation, and SGT166 pretreatment significantly reduced ROS production, whereas SGT showed a negligible blocking of ROS production (Fig. 5). For this reason, the protective effects of SGT and SGT166 against toxicant-induced disruption of MMP in SH-SY5Y cells were evaluated using JC-1 staining. As shown in Fig. 6, treatment with $50 \mu \mathrm{M} \mathrm{H}_{2} \mathrm{O}_{2}$ or $50 \mu \mathrm{M}$ etoposide decreased red fluorescence and increased green fluorescence in the cells, which indicated MMP loss. Particularly, $250 \mu \mathrm{g} / \mathrm{mL}$ SGT166 protected against MMP disruption induced by all toxicants in SH-SY5Y cells, which showed approximately three-times significantly greater activity than that of SGT.

\section{Discussion}

Lactobacillus strains are components of the intestinal microflora in humans and known to be associated with the health of the host $[22,23]$. It has also been reported that Lactobacillus strains exhibit glycosidase activity and have a critical role in the intestinal hydrolysis of various plant glycosides [24]. Cho et al. reported on the hydrolysis of daidzin and genistin to their respective aglycones, daidzein and genistein, by the Lactobacillus strains. The concentrations of daidzein and genistein increased by 910 and $830 \%$, respectively, following fermentation [25]. As a major component of glycyrrhizae radix, glycyrrhizin is often referred to by chemists as glycyrrhizic acid. Glycyrrhizin is metabolized to glycyrrhizic acid, a major

Table 2 Amounts of the seven compounds in SGT and fermented SGTs (FSGTs)

\begin{tabular}{|c|c|c|c|c|c|c|c|}
\hline \multirow{2}{*}{$\begin{array}{l}\text { Sample } \\
\text { (Lactobacillus strains) }\end{array}$} & \multicolumn{7}{|c|}{ Amounts (mg/g) } \\
\hline & Liquiritin & Ginsenoside $\mathrm{Rg}^{1}$ & Liquiritigenin & Glycyrrhizin & Atractylenolide III & Atractylenolide II & Atractylenolide I \\
\hline SGT & 2.818 & N.D. ${ }^{a}$ & 0.327 & 1.631 & 0.534 & 0.117 & 0.060 \\
\hline SGT127 (L. rhamnosus) & 0.035 & N.D. & 4.709 & 4.884 & 0.696 & 0.123 & 0.079 \\
\hline SGT129 (L. zeae) & 0.036 & N.D. & 4.718 & 5.720 & 0.695 & 0.130 & 0.079 \\
\hline SGT144 (L. rhamnosus) & 0.034 & N.D. & 4.662 & 5.808 & 0.678 & 0.119 & 0.079 \\
\hline SGT150 (L. acidophilus) & 0.031 & N.D. & 4.565 & 5.486 & 0.690 & 0.117 & 0.076 \\
\hline SGT162 (L. fermentum) & 0.061 & N.D. & 4.499 & 5.044 & 0.673 & 0.123 & 0.079 \\
\hline SGT166 (L. plantarum) & 0.034 & N.D. & 4.904 & 6.186 & 0.694 & 0.124 & 0.082 \\
\hline SGT217 (L. acidophilus) & 0.031 & N.D. & 4.678 & 6.050 & 0.645 & 0.122 & 0.078 \\
\hline SGT341 (L. helveticus) & 0.031 & N.D. & 4.472 & 5.920 & 0.696 & 0.126 & 0.079 \\
\hline
\end{tabular}

${ }^{\mathrm{a} N . D .,}$ Not detected 
Table 3 Conversion rate of detected six compounds in SGT116

\begin{tabular}{lclllll}
\hline Sample (strains) & \multicolumn{3}{l}{ Conversion rate (\%) } & & & \\
\cline { 2 - 7 } & Liquiritin & Liquiritigenin & Glycyrrhizin & Atractylenolide III & Atractylenolide II & Atractylenolide I \\
\hline SGT & 100.00 & 100.00 & 100.00 & 100.00 & 100.00 & 100.00 \\
SGT166 (Lactobacillus plantarum) & -98.79 & 1499.69 & 379.28 & 129.96 & 105.98 & 136.67 \\
\hline
\end{tabular}

physiologically active ingredient, by $\beta$-D-glucuronidase in vivo [26]. The carboxyl group in glycyrrhizin is representative of the acidic substances produced by Lactobacillus strains during fermentation in a reduced $\mathrm{pH}$ environment [27]. In our previous study, conversion from liquiritin to liquiritigenin and an increase in glycyrrhizin contents caused by Lactobacillus bacteria was observed in fermented Yijin-tang, a traditional prescription [28]. However, the greatest fermentation efficacy was observed for YJ221 fermented by L. brevis, not for YJ166 fermented by $L$. plantarum. These results suggest that the optimal Lactobacillus strain could be applied to achieve conversion of specific phytochemicals in herbal medicine and that L. plantarum 166 might be the optimal Lactobacillus strain for fermenting SGT.

Oxidative stress is involved in cell proliferation, differentiation, and survival by activating signaling pathways; however, persistent or high levels of oxidative stress cause neurotoxicity in neurodegenerative diseases $[15,29]$. Mitochondrial permeability is associated with cell toxicity, oxidative damage, and apoptosis. ROS causes mitochondrial dysfunction through mitochondrial matrix swelling and outer membrane rupture, which ultimately triggers ROS-dependent mitochondrial apoptosis $[19,20]$. Aggregated JC-1 exhibits red fluorescence in healthy mitochondria, whereas the monomeric form is characterized by green fluorescence when mitochondria are depolarized during apoptotic cell death. The intensity ratio of red/green fluorescence reflects the degree of intact mitochondria. In this study, the pretreatment of
SGT had a very low preventive effect against the MMP loss caused by $\mathrm{H}_{2} \mathrm{O}_{2}$ or etoposide even though some activity was observed at high concentrations $(500 \mu \mathrm{g} / \mathrm{mL})$ relative to that of toxicant-treated cells. In contrast, SGT166 pretreatment strongly prevented the loss of MMP by $\mathrm{H}_{2} \mathrm{O}_{2}$ or etoposide, which demonstrates that the changed phytochemicals in SGT166 by fermentation are involved in improving neuroprotective effect.

In the HPLC analysis of SGT and SGT166, liquiritigenin, glycyrrhizin, and atractylenolide I-III were increased by Lactobacillus fermentation. Previous studies have reported that an ethanol extract of Glycyrrhizae radix containing liquiritigenin and glycyrrhizin prevented amyloid beta-induced neuronal cell death by interfering with ROS [30]. Atractylodis rhizome and its active components, atractylenolide I and III, also have been reported to exert a neuroprotective effect against neuronal apoptosis [31-33]. Recently, atractylenolide I was shown to exert significant anti-neuroinflammatory activities in vitro and in a Parkinson's disease model in vivo [34]. This evidence supports the hypothesis that an increase in active compounds caused by fermentation of SGT might strongly contribute to the neuroprotective effect in SH-SY5Y human neuroblastoma. In addition, these results support that inhibiting ROS generation may be one of the neuroprotective actions of SGT166.

\section{Conclusion}

This study demonstrated that Lactobacillus fermentation enhanced the specific effects of an herbal medicine,
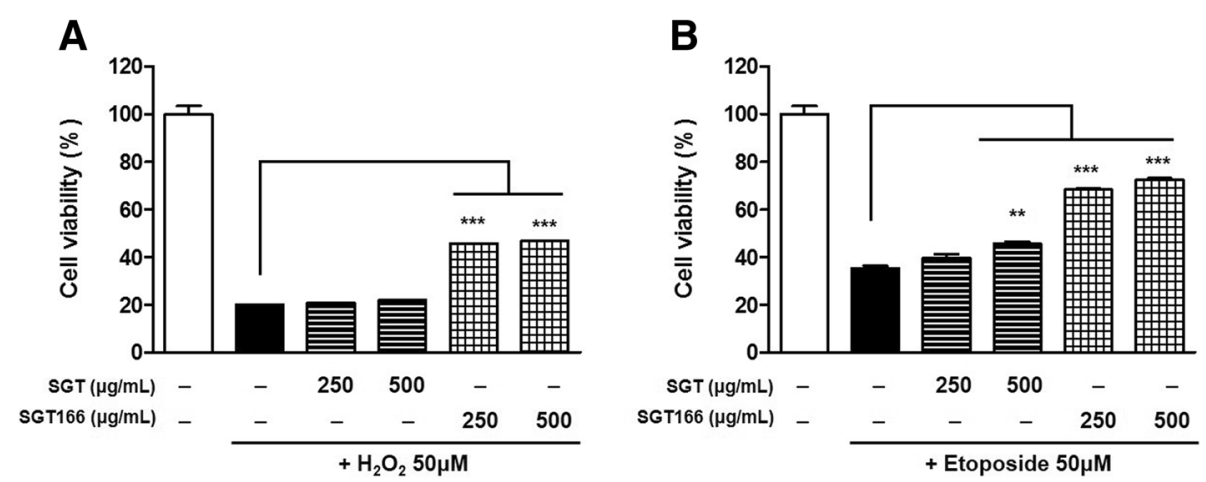

Fig. 4 Neuroprotective effect of SGT and SGT166 against toxicant-induced SH-SY5Y cell loss. Cells were pretreated with SGT or SGT166 for 6 h, and then separately exposed to $50 \mu \mathrm{M} \mathrm{H}_{2} \mathrm{O}_{2}$ (a) and $50 \mu \mathrm{M}$ etoposide (b) for $24 \mathrm{~h}$. Cell viability was determined by CCK analysis and the results are expressed as the percentages of viable cells compared to the cells untreated with any toxicant. The values shown are means \pm SE $(n=5)$. ${ }^{* *} P<0.01$ and ${ }^{* * *} P<0.001$ versus the cells treated with toxicant only 

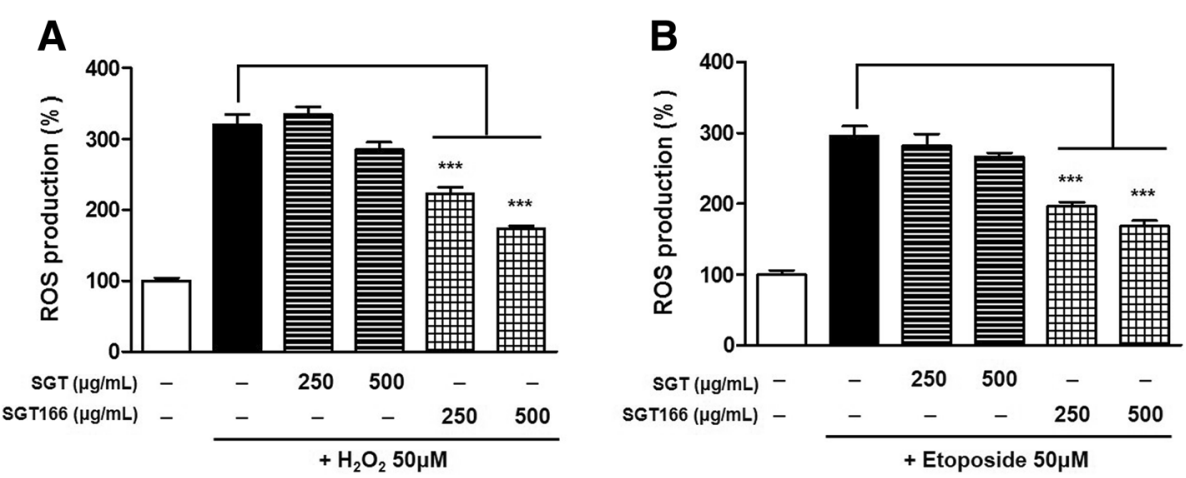

Fig. 5 The inhibitory effects of SGT and SGT166 against toxicant-induced ROS production in SH-SY5Y cells. Total ROS levels were measured using the $\mathrm{H}_{2}$-DCFDA method. Cells were pretreated with SGT or SGT166 for $6 \mathrm{~h}$, and then labeled with $50 \mu \mathrm{M} \mathrm{H} \mathrm{H}_{2}$-DCFDA for 30 min. Cells were separately exposed to $50 \mu \mathrm{M} \mathrm{H}_{2} \mathrm{O}_{2}$ (a) and $50 \mu \mathrm{M}$ etoposide (b), analyzed immediately using a fluorescent plate reader. The values shown are means $\pm \mathrm{SE}(n=5){ }^{* * *} P<0.001$ versus the cells treated with toxicant only

SGT. Especially, SGT166, a SGT fermented with $L$. plantarum 166, enhanced neuroprotective effects by suppressing oxidative stress and MMP loss. The enhancement was thought to be caused by conversion of phytochemicals during the fermentation. Therefore,
SGT166 is a potential candidate for treatment of neurological damage-related diseases. The study results should be complemented by investigations of the functional mechanisms of SGT166 in neuroblastoma and evaluation of the neuroprotective effect in an in vivo model.

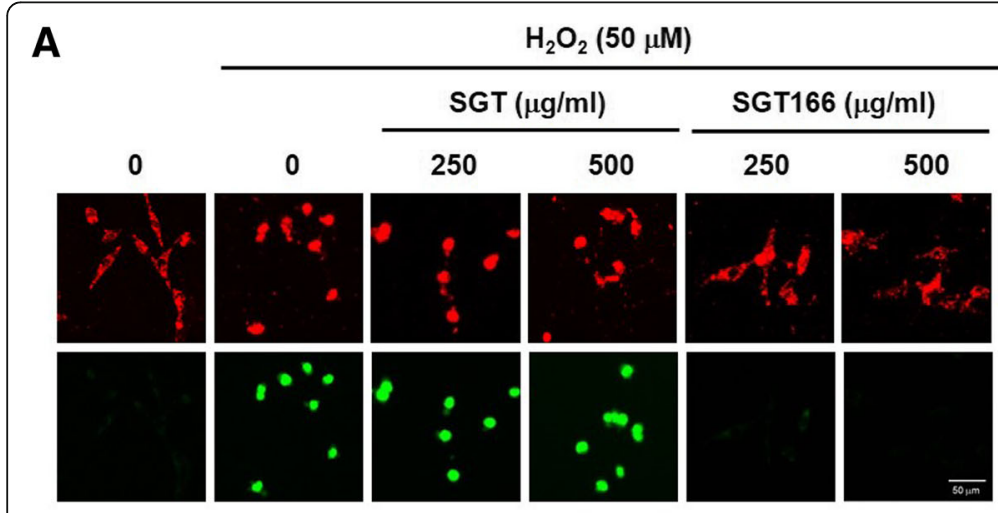

B
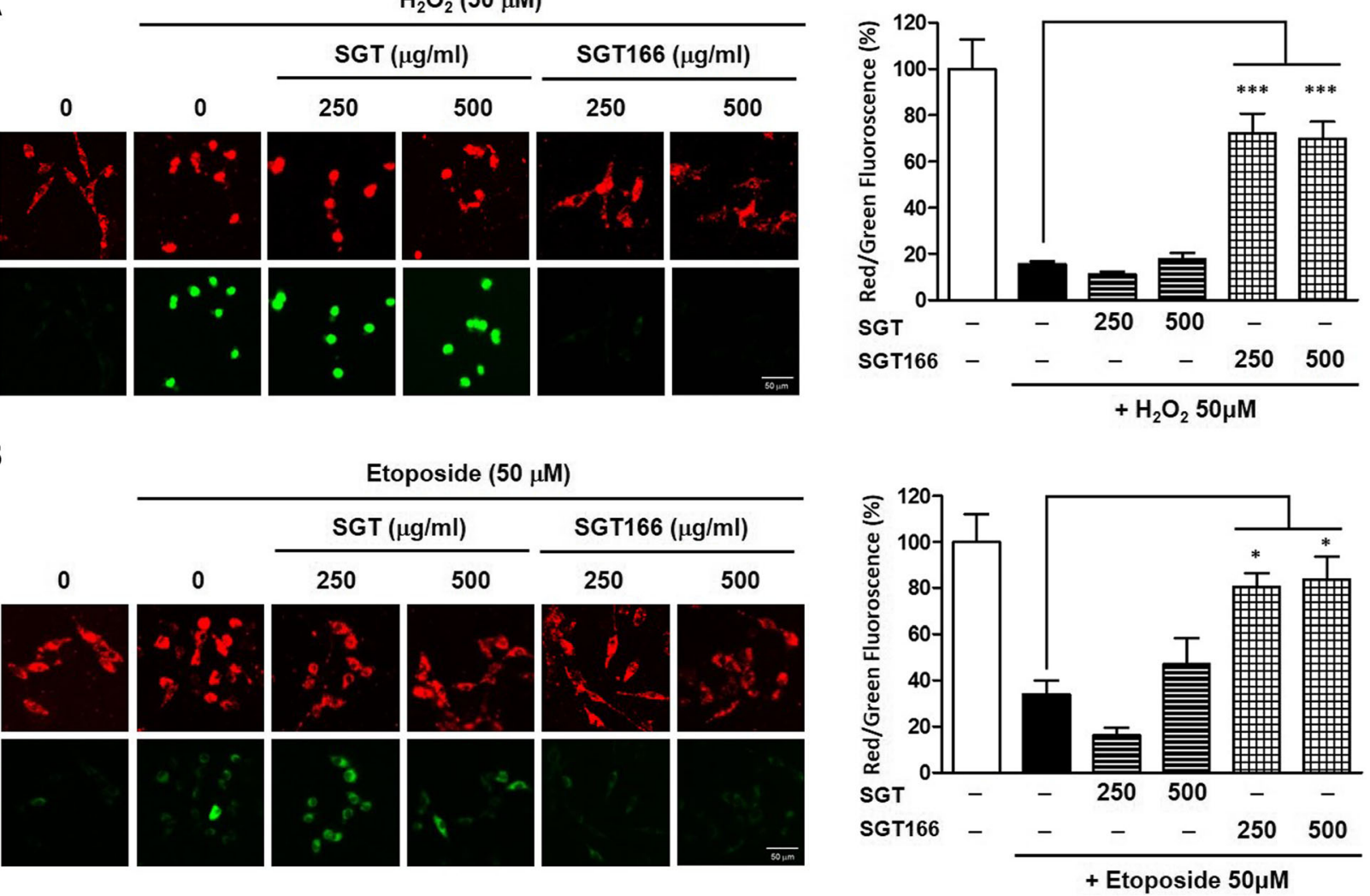

Fig. 6 The protective effects of SGT and SGT166 against toxicant-induced disruption of MMP in SH-SY5Y cells. MMP was assessed by confocal microscopy using JC-1 staining. Cells were pretreated with SGT or SGT166 for 6 h, and then separately exposed to $50 \mu \mathrm{M} \mathrm{H} \mathrm{H}_{2} \mathrm{O}_{2}(\mathbf{a})$ and $50 \mu \mathrm{M}$ etoposide (b) for $1 \mathrm{~h}$. After incubated with JC-1 for $15 \mathrm{~min}$, the cells were observed under 132x magnification. Representative images showing red fluorescence (aggregated form) and green fluorescence (monomeric form). Scale bar $=50 \mu \mathrm{m}$. The graph shows the red/green fluorescence intensity ratio quantitative analysis. The values shown are means $\pm S E(n=5) .{ }^{*} P<0.05$ and ${ }^{* * *} P<0.001$ versus the cells treated with toxicant only 


\section{Additional file}

Additional file 1: Table S1. Calibration curves, limit of detection (LOD), and limit of quantification (LOQ) of the seven compounds. Table S2. Precision (intra- and interday) and accuracy of the seven compounds. Table S3. The percent recovery of the seven compounds. Table S4. Identification of the phytochemicals in SGT and SGT166 by UPLC-ESI-MS analysis. (DOCX $31 \mathrm{~kb})$

\section{Abbreviations}

AD: Alzheimer's disease; ALS: Amyotrophic lateral sclerosis; CCK-8: Cell counting kit-8; FSGTs: Fermented SGTs; $\mathrm{H}_{2}$-DCFDA: 2', 7'dichlorodihydrofluorescein diacetate; HPLC-DAD: High-performance liquid chromatography with diode-array detection; ICH: International conference on harmonization; LC/MS: Liquid chromatography/mass spectrometry;

LOD: Linearity, limit of detection; LOQ: Limit of quantification; MMPs: mitochondrial membrane potentials; MS: Mutiple Sclerosis; PD: Parkinson's disease; RNS: Reactive nitrogen species; ROS: Reactive oxygen species; RSD: Relative standard deviation; SGT: Sagunja-tang;

TFA: Trifluoroacetic acid; YJ: Yijin-tang

\section{Acknowledgements}

Not applicable.

\section{Funding}

This research was supported by the Korea Institute of Oriental Medicine (KIOM) (Project no. K17281 and K18101) from the Ministry of Science and ICT (MSIT), Republic of Korea.

\section{Availability of data and materials}

All datasets used and analyzed during the current study are available from the corresponding author on reasonable request.

\section{Authors' contributions}

NHY and JYM designed the study and wrote the manuscript. MJG mainly carried out the experiments for the part of analysis study. HRP carried out the experiments for the part of bioassay study. YHH supported the LC/MS analysis data. All authors read and approved the final manuscript.

\section{Ethics approval and consent to participate}

Not applicable. This article is not a clinical study involving human participants and this manuscript does not contain any individual clinical data.

\section{Consent for publication}

Not applicable.

\section{Competing interests}

The authors declare that they have no competing interests.

\section{Publisher's Note}

Springer Nature remains neutral with regard to jurisdictional claims in published maps and institutional affiliations.

Received: 24 October 2017 Accepted: 25 October 2018

Published online: 28 November 2018

\section{References}

1. Liu Y, Yang J, Cai Z. Chemical investigation on Sijunzi decoction and its two major herbs Panax ginseng and Glycyrrhiza uralensis by LC/MS/MS. J Pharm Biomed Anal. 2006:41(5):1642-7.

2. Lu Y, Lin H, Zhang J, Wei J, Sun J, Han L. Sijunzi decoction attenuates 2, 4 , 6-trinitrobenzene sulfonic acid (TNBS)-induced colitis in rats and ameliorates TNBS-induced claudin-2 damage via NF-kappaB pathway in Caco2 cells. BMC Complement Altern Med. 2017;17(1):35

3. Yu W, Lu B, Zhang H, Zhang Y, Yan J. Effects of the Sijunzi decoction on the immunological function in rats with dextran sulfate-induced ulcerative colitis. Biomed Rep. 2016;5(1):83-6.
4. Zhao AG, Zhao HL, Jin XJ, Yang JK, Tang LD. Effects of Chinese Jianpi herbs on cell apoptosis and related gene expression in human gastric cancer grafted onto nude mice. World J Gastroenterol. 2002;8(5):792-6.

5. Zheng J, Liu JL, Lin MF, Wang ZF, Liu CY, Wu XH, Lin HY, Chen CF, Zheng $X M$, Chen XY. Effect of modified sijunzi decoction on the bone metabolism of adriamycin induced nephropathy rats. Zhongguo Zhong Xi Yi Jie He Za Zhi. 2013;33(10):1376-81.

6. Kang A, Guo JR, Zhang Z, Wang XL. Simultaneous quantification of ten active components in traditional Chinese formula Sijunzi decoction using a UPLC-PDA method. J Anal Methods Chem. 2014;2014:570359.

7. Trinh HT, Han SJ, Kim SW, Lee YC, Kim DH. Bifidus fermentation increases hypolipidemic and hypoglycemic effects of red ginseng. J Microbiol Biotechnol. 2007;17(7):1127-33.

8. Dai Z, Liu Y, Zhang X, Shi M, Wang B, Wang D, Huang L, Zhang X. Metabolic engineering of Saccharomyces cerevisiae for production of ginsenosides. Metab Eng. 2013;20:146-56.

9. Marazza JA, Garro MS, de Giori GS. Aglycone production by lactobacillus rhamnosus CRL981 during soymilk fermentation. Food Microbiol. 2009:26(3):333-9.

10. Ng CC, Wang CY, Wang YP, Tzeng WS, Shyu YT. Lactic acid bacterial fermentation on the production of functional antioxidant herbal Anoectochilus formosanus Hayata. J Biosci Bioeng. 2011;111(3):289-93.

11. Oh YC, Cho WK, Jeong YH, Im GY, Yang MC, Ma JY. Fermentation improves anti-inflammatory effect of sipjeondaebotang on LPS-stimulated RAW 264.7 cells. Am J Chin Med. 2012;40(4):813-31.

12. Shim KS, Kim T, Ha H, Lee KJ, Cho CW, Kim HS, Seo DH, Ma JY. Lactobacillus fermentation enhances the inhibitory effect of Hwangryun-haedok-tang in an ovariectomy-induced bone loss. BMC Complement Altern Med. 2013;13:106

13. Lee JJ, Kwon H, Lee JH, Kim DG, Jung SH, Ma JY. Fermented soshiho-tang with lactobacillus plantarum enhances the antiproliferative activity in vascular smooth muscle cell. BMC Complement Altern Med. 2014;14:78.

14. Park HR, Lee H, Park H, Cho WK, Ma JY. Fermented Sipjeondaebo-tang alleviates memory deficits and loss of hippocampal neurogenesis in scopolamine-induced amnesia in mice. Sci Rep. 2016;6:22405.

15. Hsieh $\mathrm{HL}$, Yang $\mathrm{CM}$. Role of redox signaling in neuroinflammation and neurodegenerative diseases. Biomed Res Int. 2013;2013:484613.

16. Pollari E, Goldsteins G, Bart G, Koistinaho J, Giniatullin R. The role of oxidative stress in degeneration of the neuromuscular junction in amyotrophic lateral sclerosis. Front Cell Neurosci. 2014;8:131.

17. Padurariu M, Ciobica A, Lefter R, Serban IL, Stefanescu C, Chirita R. The oxidative stress hypothesis in Alzheimer's disease. Psychiatr Danub. 2013;25(4):401-9.

18. Murakami S, Miyazaki I, Sogawa N, Miyoshi K, Asanuma M. Neuroprotective effects of metallothionein against rotenone-induced myenteric neurodegeneration in parkinsonian mice. Neurotox Res. 2014;26(3):285-98.

19. Liu CY, Lee CF, Wei YH. Role of reactive oxygen species-elicited apoptosis in the pathophysiology of mitochondrial and neurodegenerative diseases associated with mitochondrial DNA mutations. J Formos Med Assoc. 2009;108(8):599-611.

20. Akopova OV, Kolchynskayia LY, Nosar VY, Smyrnov AN, Malisheva MK, Man'kovskaia YN, Sahach VF. The effect of permeability transition pore opening on reactive oxygen species production in rat brain mitochondria. Ukr Biokhim Zh (1999). 2011;83(6):46-55.

21. Park HR, Lee $H$, Park H, Jeon JW, Cho WK, Ma JY. Neuroprotective effects of Liriope platyphylla extract against hydrogen peroxide-induced cytotoxicity in human neuroblastoma SH-SY5Y cells. BMC Complement Altern Med. 2015;15:171.

22. Castro JJ, Gomez A, White B, Loften JR, Drackley JK. Changes in the intestinal bacterial community, short-chain fatty acid profile, and intestinal development of preweaned Holstein calves. 2. Effects of gastrointestinal site and age. J Dairy Sci. 2016;99(12):9703-15.

23. Masood MI, Qadir MI, Shirazi JH, Khan IU. Beneficial effects of lactic acid bacteria on human beings. Crit Rev Microbiol. 2011;37(1):91-8.

24. Michlmayr H, Schumann C, da Silva NM, Kulbe KD, del Hierro AM. Isolation and basic characterization of a beta-glucosidase from a strain of lactobacillus brevis isolated from a malolactic starter culture. J Appl Microbiol. 2010:108(2):550-9.

25. Cho CW, Jeong HC, Hong HD, Kim YC, Choi SY, Kim K, Ma JY, Lee YC. Bioconversion of isoflavones during the fermentation of Samso-Eum with lactobacillus strains. Biotechnol Bioprocess Eng. 2012;17(5):1062-7. 
26. Glavac NK, Kreft S. Excretion profile of glycyrrhizin metabolite in human urine. Food Chem. 2012;131(1):305-8.

27. Li J, Liu S, Wang J, Li J, Li J, Gao W. Gene expression of glycyrrhizin acid and accumulation of endogenous signaling molecule in Glycyrrhiza uralensis Fisch adventitious roots after Saccharomyces cerevisiae and Meyerozyma guilliermondii applications. Biotechnol Appl Biochem. 2017;64(5):700-11.

28. Yang HJ, Yim NH, Lee KJ, Gu MJ, Lee B, Hwang YH, Ma JY. Simultaneous determination of nine bioactive compounds in Yijin-tang via highperformance liquid chromatography and liquid chromatographyelectrospray ionization-mass spectrometry. Integr Med Res. 2016;5(2):140-50.

29. Dai DF, Chiao YA, Marcinek DJ, Szeto HH, Rabinovitch PS. Mitochondrial oxidative stress in aging and healthspan. Longev Healthspan. 2014;3:6.

30. Lee HK, Yang EJ, Kim JY, Song KS, Seong YH. Inhibitory effects of Glycyrrhizae radix and its active component, isoliquiritigenin, on Abeta(2535)-induced neurotoxicity in cultured rat cortical neurons. Arch Pharm Res. 2012;35(5):897-904.

31. Gao Q, Ji ZH, Yang Y, Cheng R, Yu XY. Neuroprotective effect of Rhizoma Atractylodis macrocephalae against excitotoxicity-induced apoptosis in cultured cerebral cortical neurons. Phytother Res. 2012;26(4):557-61.

32. Ji ZH, Liu C, Zhao H, Yu XY. Neuroprotective effect of biatractylenolide against memory impairment in D-galactose-induced aging mice. J Mol Neurosci. 2015;55(3):678-83.

33. Liu C, Zhao H, Ji ZH, Yu XY. Neuroprotection of atractylenolide III from Atractylodis macrocephalae against glutamate-induced neuronal apoptosis via inhibiting caspase signaling pathway. Neurochem Res. 2014;39(9):1753-8.

34. More S, Choi DK. Neuroprotective role of Atractylenolide-l in an in vitro and in vivo model of Parkinson's disease. Nutrients. 2017;9(5):451.

Ready to submit your research? Choose BMC and benefit from:

- fast, convenient online submission

- thorough peer review by experienced researchers in your field

- rapid publication on acceptance

- support for research data, including large and complex data types

- gold Open Access which fosters wider collaboration and increased citations

- maximum visibility for your research: over $100 \mathrm{M}$ website views per year

At $\mathrm{BMC}$, research is always in progress.

Learn more biomedcentral.com/submissions 